\title{
Study of the prototype mechanism of height adjustment of the bed in hospital bed
}

\author{
Ireneusz Malujda ${ }^{1}$, Dominik Wilczyński ${ }^{1,{ }^{*}}$, Krzysztof Talaśka ${ }^{1}$, Dominik Wojtkowiak ${ }^{1}$, \\ Maciej Szulc ${ }^{2}$ \\ ${ }^{1}$ Poznan University of Technology, Chair of Basics of Machine Design, Piotrowo Str. 3, Poland \\ ${ }^{2}$ MSprojekt Maciej Szulc, Strumykowa 8 Str., Opalenica, Poland
}

\begin{abstract}
The presented kinematic system of the mechanism is an effect of a design process taking into an account design objectives specified by the medical staff in a hospital. In order to meet these requirements, a kinematic analysis of the mechanism was performed with the objective of finding a connection between various loads of the bed deck and the minimum force needed to drive the height adjustment mechanism. The next step was the validation process, performed by building the prototype of the studied machine on which the empirical tests were completed. During testing, the drive force of the mechanism was measured for various values and distribution of the load. The obtained results were compared with the results of the simulation analysis. This made it possible to reach the final research goal, which was to determine how to place all the mechanism parts in order to achieve the lowest possible drive force and fulfil all design objectives simultaneously.
\end{abstract}

Keywords: hospital bed, height adjustment mechanism, kinematic analysis

\section{Introduction}

Hospital beds used by health care professionals are operated manually in most cases, which requires some physical effort $[1,4]$. In the last few years there has been the development of their design dictated by the need to improve the comfort of patients and hospital workers [1, 5 , 10]. It is mainly about minimising risks caused by, among others, mistakes and indisposition of the medical personnel occurring during the transport of patients in lifethreatening conditions $[5,6]$. Solutions include, among others, automation of bed movement by means of motorised devices, for both functional and transport purposes [2]. In addition, it concerns devices that will allow the transfer of a patient from one bed to another, or from a bed to a wheelchair $[3,4,7]$, devices providing periodic, automatic change of a lying patient's position in order to prevent the emergence of bedsores $[8,9]$, and advanced, automated wheelchairs [11-13]. The technical development of this kind of devices and their application in the hospital environment are facing many difficulties and limitations [1].

This work presents a design solution for the hospital bed height adjustment mechanism developed within the objectives related to the requirements set for one of the hospitals in

\footnotetext{
*Corresponding author: dominik.wilczynski@put.poznan.pl

Reviewers: Robert Grega, Milan Nad'
} 
Poznan. The main objective for the mechanism design is to provide comfort and safety for the patient as well as for the medical personnel when performing diagnostic tests.

\section{Design objectives}

Based on the interview with the medical staff of the cardiac surgery ward, design objectives for a specialized mobile set consisting of a hospital bed and a medical equipment stand were formulated. One of the important elements of this set is the bed deck height adjustment mechanism. bed:

The following geometric parameters and design objectives have been assumed for the

- overall bed dimensions in the bed deck plane: 2090 x $1000 \mathrm{~mm}$,

- maximum bed height: $1200 \mathrm{~mm}$,

- adjustable bed deck height in the $500 \div 800 \mathrm{~mm}$ range,

- lifting mechanism drive started manually by staff,

- ability to introduce a probe (head of a diagnostic device) under the bed deck, in order to perform diagnostic tests without having to move (rotate) the patient,

- back angle adjustment in the $0 \div 70^{\circ}$ range started manually by personnel,

- ability to measure the weight of the patient,

- ability to attach a medical equipment stand,

- large, rotating wheels with braking function,

- bumper wheels in extreme points of the design,

- handles for easy manoeuvring,

- protective railings,

- design guards.

Meeting the specified objectives and the necessary adjustment functionality required a kinematic analysis and designing the adjustment mechanism for the bed deck on its basis.

\section{Design and operating principle of the bed deck height adjustment mechanism}

The kinematic diagram of the bed top height adjustment mechanism is shown in Figure 1a and $b$.

Hospital bed deck height adjustment mechanism 12 (upper frame) consists of two systems of drive arms 4 and 10 and 18 and 22 coupled with kinematic pairs turning around the $\mathrm{z}$ axes, 11 and 21, operating as a scissors lifting system (fig. 1). Drive arms systems 4 and 10 , and 18 and 22 connect the base frame 6 with the bed deck 12 through kinematic pairs turning around the $\mathrm{z}$ axes, 5 and 13 and 17 and 23, and are integrated in the extreme positions looking along the longer side of the base frame 6 and table-top 12 (along the $x$ axis). The drive mechanism 1 is embedded at the height of the base frame 6 and constitutes an active sliding kinematic pair that sets into angular motion one of the two pairs of drive arms 4 and 10, and indirectly the second pair of the drive arms 18 and 22 by means of drive transmission beam 9 (fig. 1). Movement in the vertical direction (along the y axis) of bed deck 12 is provided by guide sleeves 14 and 19, and guide rails 15 and 20. Guide sleeves 14 and 19 are attached to the base frame 6 and guide rails 15 and 20 are attached to bed deck 12 (fig. 1).

The drive mechanism 1 can be any active sliding kinematic pair with one degree of freedom connected to the beams of the base frame 24 with a kinematic pair turning around the $\mathrm{z}$ axis 2 . The beams of the base frame 24 constitute an element of the base frame structure 6 . 
In order to select geometric parameters of individual segments of the mechanism, a kinematic analysis discussed in the further part of this work was performed.

a)

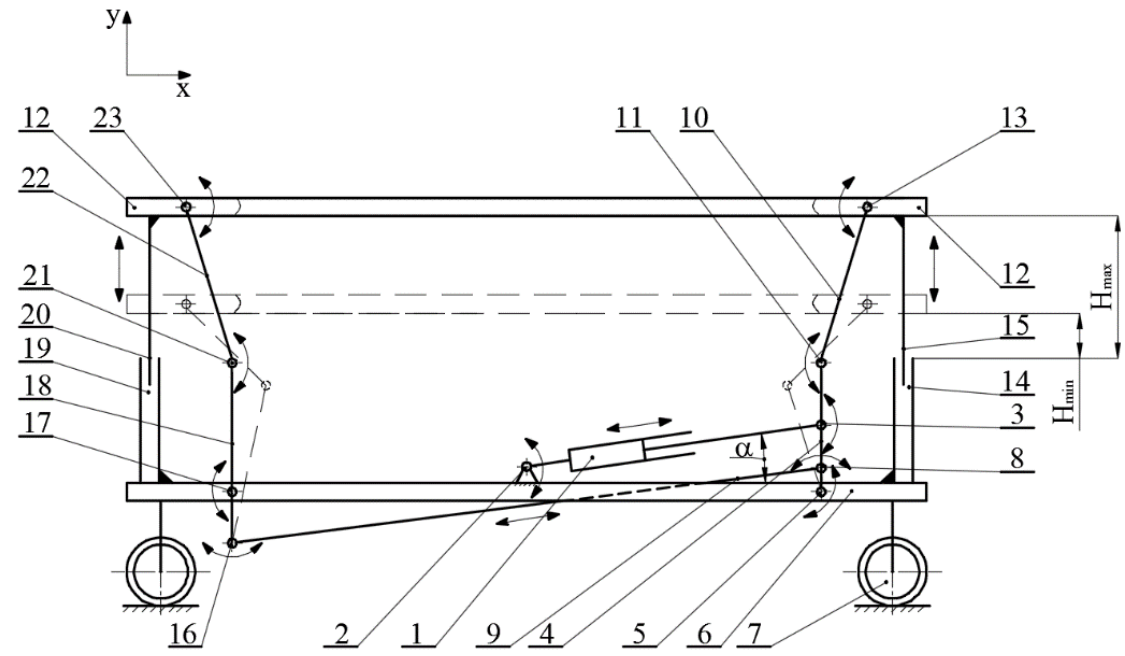

b)

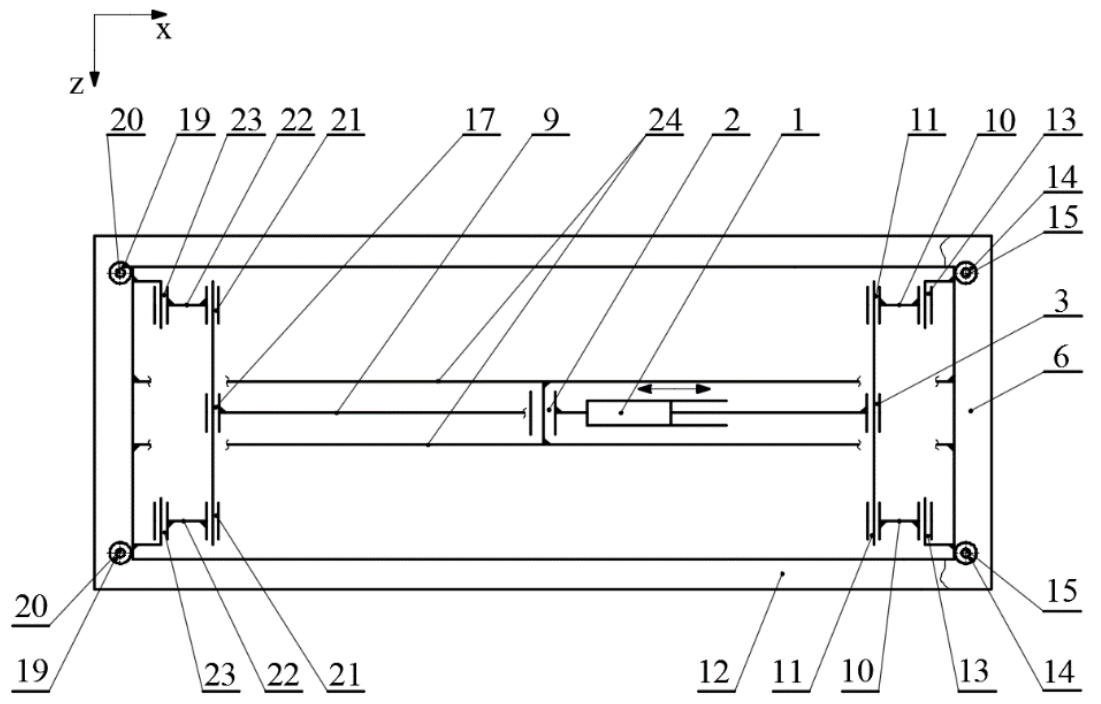

Fig. 1. Kinematic diagram of the bed top height adjustment mechanism in a hospital bed:

a) view in the xy plane, b) view from the top in the xz plane, where: 1 - drive mechanism, 2 - turning kinematic pair, 3 - turning kinematic pair, 4 - drive arm, 5 - turning kinematic pair, 6 - base frame, 7 - wheel set, 8 - turning kinematic pair, 9 - drive transmission beam, 10 - drive arms, 11 turning kinematic pairs, 12 - upper frame, 13 - turning kinematic pair, 14 - guide bush, 15 - guide rail, 16 - turning kinematic pair, 17 - turning kinematic pair, 18 - drive arm, 19 - guide bush, 20 - guide rail, 21 - turning kinematic pair, 22 - drive arms, 23 - turning kinematic pair, 24 - horizontal axis, 25 - horizontal axis, 26 - base frame beams 


\section{Kinematic analysis of the bed deck height adjustment mechanism and its results}

One of the main purposes of performing a kinematic analysis was to determine the angle $\alpha$ between the axis of the actuator, and the $x z$ plane of the base frame (fig. 1). The value of this angle has a significant influence on the initial value of the force needed to start the bed deck height adjustment mechanism (lowest bed deck position $\mathrm{H}_{\text {min }}$, looking along the y axis, fig. 1). The numerical simulation was used to specify the value of the angle $\alpha$ determining the lowest possible value of the forces with regards to the actuator that starts the process of lowering and raising the bed deck. It is important that the initial angle $\alpha$ has such a value that in the upper position of the bed deck there is enough space underneath to make it possible to manoeuvre medical diagnostic instruments. Simulations were made also at specific bed deck load values (assuming different load values depending on the patient's weight). The kinematic model (fig. 2) of the bed deck height adjustment mechanism was built using SolidWorks in order to seek the specific values of the geometric parameters: A, B, C, D, E, F, G, H, I, J, $\alpha$ (fig. 2). It was assumed that the drive mechanism would make use of a hydraulic actuator, whose sliding movement kicks in as a result of starting a foot-powered pump integrated with the actuator in a single body. The simulation model takes into account the actual geometric parameters of the drive, $\mathrm{A}_{\min }$ - initial length, $\mathrm{A}_{\max }$ - final length with a fully extended rod.

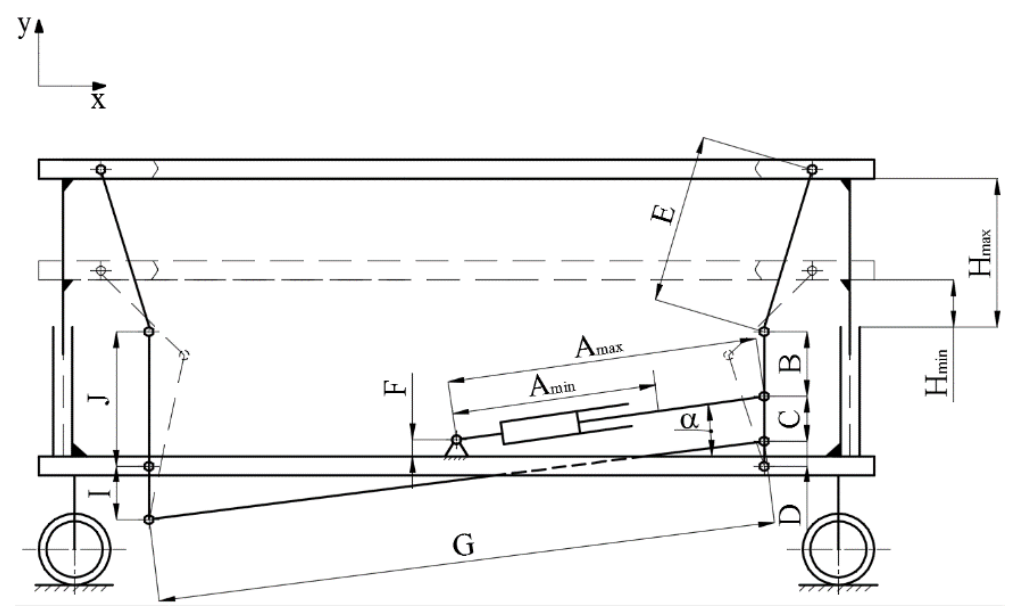

Fig. 2. Kinematic diagram of bed deck height adjustment mechanism with desired geometric parameters of individual parameters

The analysis includes the following design limitations and project assumptions:

- the distance between the ground and the bottom surface of the base frame is $200 \mathrm{~mm}$ (wheel diameter $150 \mathrm{~mm}+$ wheel stud length $50 \mathrm{~mm}$ ),

- hydraulic actuator stroke $-300 \mathrm{~mm}$, maximum strength $-10000 \mathrm{~N}$.

- bed deck height adjustment in the $500 \div 800 \mathrm{~mm}$ range, which means that the height $\mathrm{H}$ should be adjusted in the $300 \div 600 \mathrm{~mm}$ range.

- to prevent the mechanism from blocking, and to obtain free space under the bed deck where the patient is lying, at the maximum height of the bed, the angle between the drive arms 10 and 22 (fig. 1), and the vertical y axis should be within the $15^{\circ} \div 30^{\circ}$ range,

- angle $\alpha$ at zero actuator extension should be greater than $0^{\circ}$ so that the actuator's body is not located below the xz plane of the structural profiles of the base frame 6 (fig. 1b). 
Figure 3 presents a sample simulation result in the form of the characterization of the changes in the value of the force required to start the bed deck height adjustment mechanism. The characterization applies to changes in the value of the force needed to raise the bed deck for the angle $\alpha=7.8^{\circ}$ and the bed deck load 12 (fig. 1a) corresponding to the patient's weight of $86 \mathrm{~kg}$.

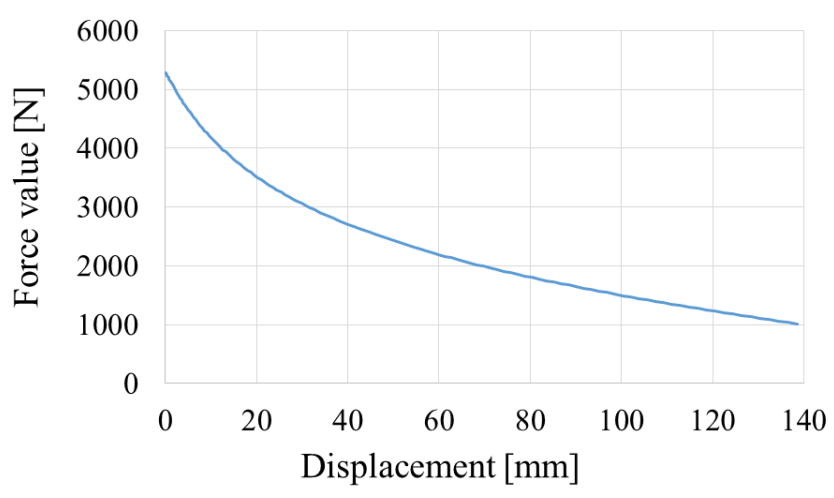

Fig. 3. Characterization of the changes in the value of the force required to start the mechanism in the function of displacement obtained on the basis of the numerical simulation $\alpha=7.8^{\circ}$ and $\mathrm{m}=86 \mathrm{~kg}$

The next figure shows the characterization of the changes of the force needed to start the bed deck adjustment mechanism for the angle $\alpha=11.7^{\circ}$ and the bed deck load 12 (fig. 1a) corresponding to the patient's weight of $86 \mathrm{~kg}$.

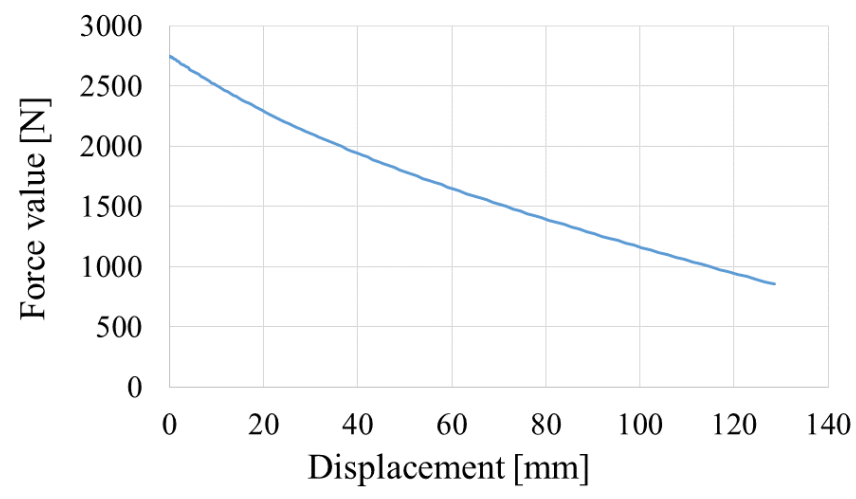

Fig. 4. Characterization of the changes in the value of the force required to start the mechanism in the function of displacement obtained on the basis of the numerical simulation $\alpha=11.7^{\circ}$ and $\mathrm{m}=86 \mathrm{~kg}$

As is apparent from the above characterizations, as you increase the initial value of the angle $\alpha$ from $\alpha=7.8^{\circ}$ to $\alpha=11.7^{\circ}$, the initial value of the force required to start the mechanism is reduced from $\mathrm{F}=5276 \mathrm{~N}$ to $\mathrm{F}=2583 \mathrm{~N}$.

In the next stage of the design process the bed prototype was built, and subsequently the verification of the simulation results was performed by comparing them with the results of measurements of the force changes obtained from the study of the bed mechanism prototype. 


\section{Building and testing the prototype}

The kinematic analysis of the bed height adjustment mechanism formed the basis for determining the geometric parameters of the kinematic chain of the mechanism A, B, C, D, E, F, G, H, I, J, $\alpha$ (fig. 2), which are discussed in the previous section. This made it possible to build the bed prototype using the lifting mechanism (fig. 1 and 2) and to study its functional characteristics.

The view of the base frame with attached vertical guide rails, which in turn are connected to the upper frame is shown in Figure 5.

The build-phase bed prototype with the mounted mechanism for lifting the bed deck with the kinematic system as shown in Figure 1 and 2 is presented below (fig. 6).

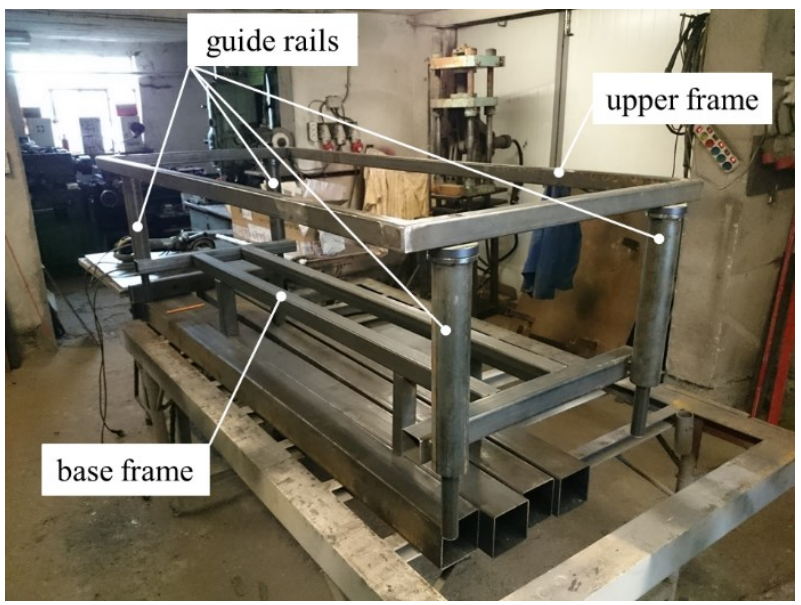

Fig. 5. Bed frame of the research and prototyping station at one of the build stages

The bed with the functional bed deck height adjustment mechanism is presented in Figure 6.

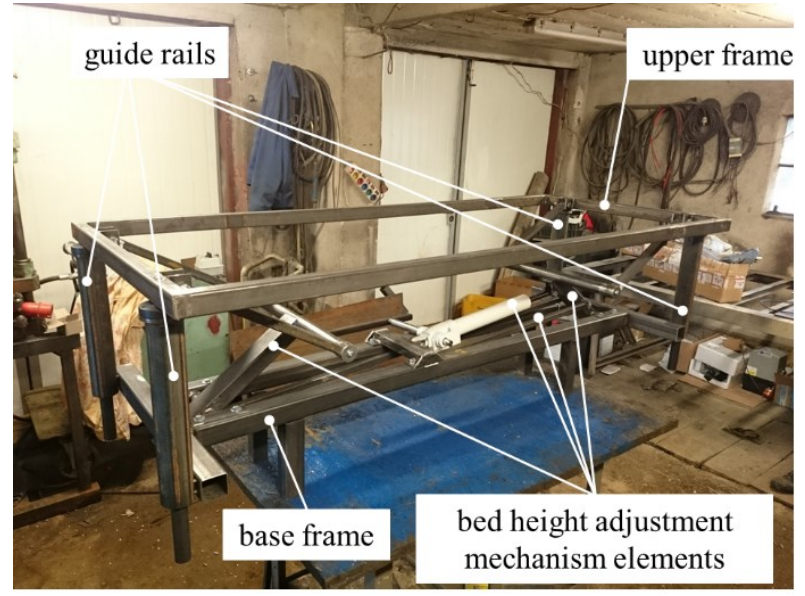

Fig. 6. Bed frame with the enclosed bed deck height adjustment mechanism 
Figure 7 shows the partially elevated bed deck and partially extended piston rod of the hydraulic actuator.

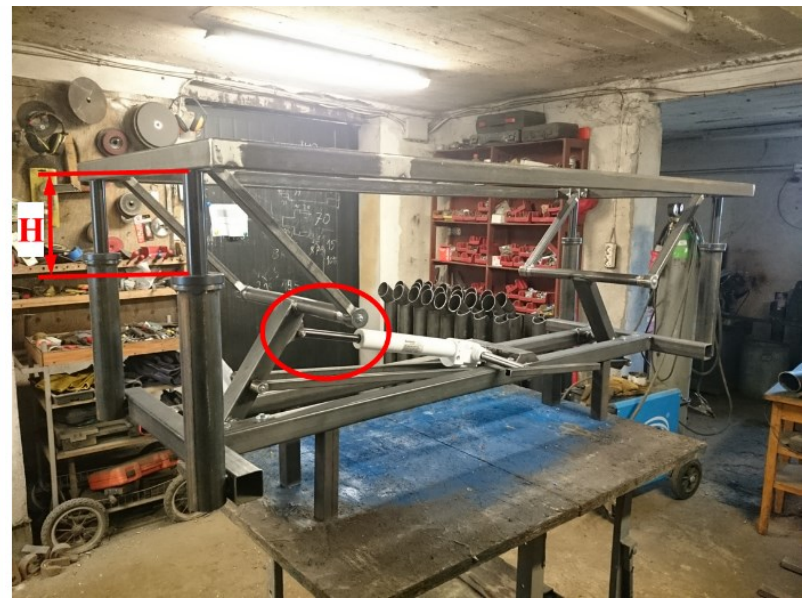

Fig. 7. General view of the bed height adjustment mechanism while in operation - piston rod of the actuator partially extended, bed deck raised to height $\mathrm{H}$

The drive mechanism consists of a hydraulic actuator by Bansbach, started with a footpowered pump enclosed in a single body. The maximum axial force with which the actuator can interact with the powered mechanism is $\mathrm{F}=10 \mathrm{kN}$ and the maximum extension of the rod is $\mathrm{s}=300 \mathrm{~mm}$ (fig. 8). The following figure shows the overall view of the actuator.

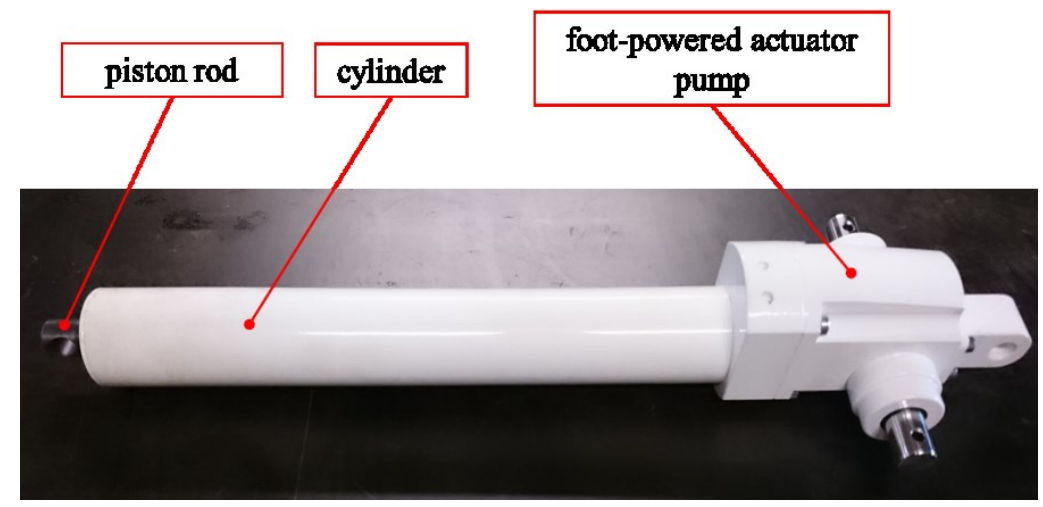

Fig. 8. Overall view of the hydraulic cylinder powering the bed deck height adjustment mechanism in a hospital bed

The mechanism was studied with regards to determining the force F necessary to start it and the results were compared to the results of the numerical analysis. Bearing in mind that the values of the force $F$ necessary to start the mechanism obtained by means of the numerical simulation can significantly deviate from the value of the actual forces, the prototype was built using a hydraulic cylinder with the highest possible drive force. Figure 9 shows the research station consisting of the bed prototype, the force sensor and the displacement sensor. 


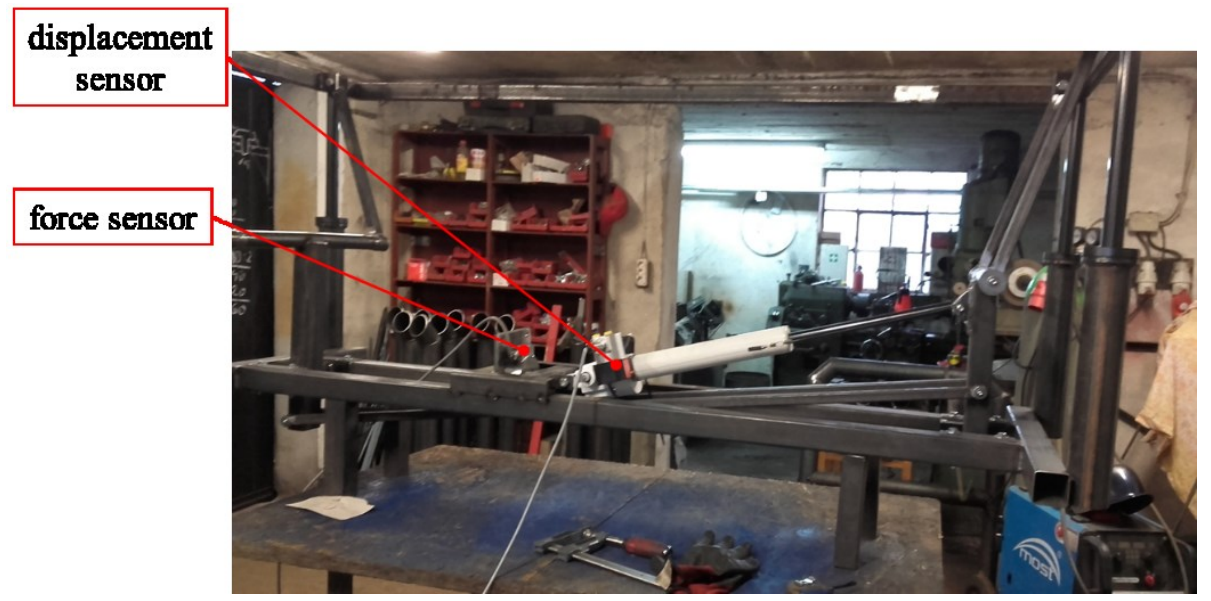

Fig. 9. View of the bed frame when measuring the force needed to power the bed deck height adjustment mechanism

The studies of the force $\mathrm{F}$ needed to start the bed deck height adjustment mechanism were performed for two values of the angle $\alpha=7.8^{\circ}$ and $11.7^{\circ}$. This formed the foundation for comparing the simulation results with the actual results of prototype testing.

Figure 10 shows the characterization comparison for the changes of the force $F$ acting on the axis of the hydraulic actuator obtained from the simulation and the experimental studies for the angle $\alpha=7.8^{\circ}$ and the bed deck load 12 (fig. 1a) corresponding to the load with the patient's weight $\mathrm{m}=86 \mathrm{~kg}$.

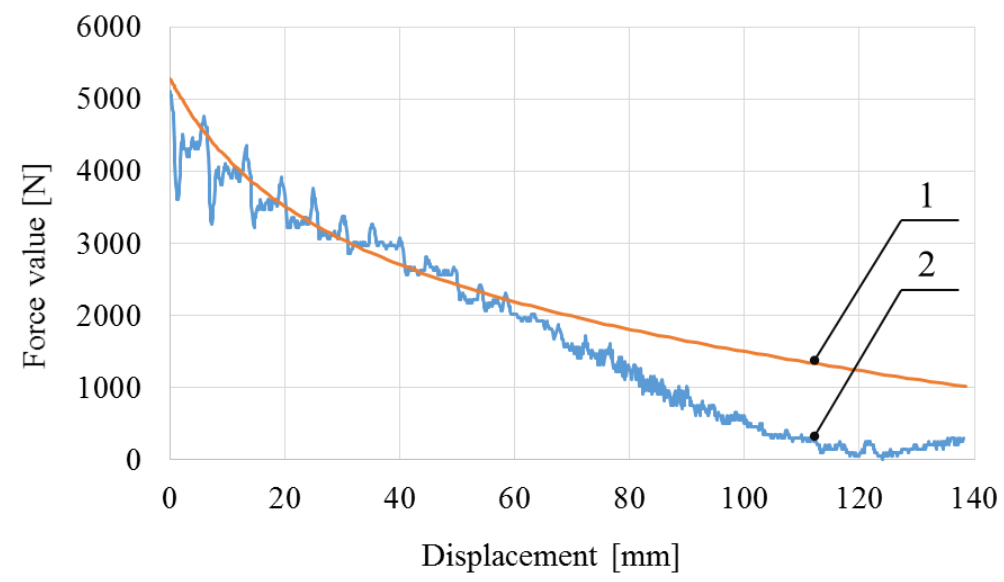

Fig. 10. Characterization comparison of the changes of the force $F$ acting on the axis of the hydraulic actuator rod needed to power the bed deck adjustment mechanism for the angle

$\alpha=7.8^{\circ}$ and the load corresponding to the patient's weight $\mathrm{m}=86 \mathrm{~kg}$, where: 1 - characterization obtained from simulation, 2 - characterization obtained from testing

The maximum force $\mathrm{F}$ obtained from the prototype tests is $5107 \mathrm{~N}$ whereas from the numerical analysis it is $5276 \mathrm{~N}$.

Surges in changes of the force obtained from the tests are due to the surge-prone nature of the drive mechanism, which results from the foot pumping of the oil to the cylinder chamber during the piston rod extension. Pumping is done by means of the angular rotation 
of the pump shaft, at which point the oil is pumped to the cylinder chamber, which in turn causes the rod to get extended and power the mechanism. After the pumping cycle movement is complete, the piston rod stops, which is visible as a decrease in the value of the force. The numerical simulation assumes a continuous interaction of the force with the mechanism, which is why its course is smooth.

As the value of the angle $\alpha$ increases, the force F decreases, which follows from both the numerical simulations and the actual results of the prototype tests. The value of the force obtained from the simulation falls slightly faster, which can be explained by not taking into account drag forces in the numerical model (e.g. friction in individual turning kinematic pairs).

Figure 11 presents the characterization comparison of the changes of the force $(F)$, taken from the simulation and the actual prototype tests, for the angle $\alpha=11.7^{\circ}$ and the bed deck load 12 (fig. 1a) with the patient's weight $\mathrm{m}=86 \mathrm{~kg}$.

As a result of the change in the minimum value of the angle $\alpha$ from initial $7.8^{\circ}$ (corresponding to the minimum bed deck height) to $11.7^{\circ}$ (minimum bed deck height) with the bed deck load $\mathrm{m}=86 \mathrm{~kg}$, there is a $36 \%$ decline in the value of the force $\mathrm{F}$ from $5107 \mathrm{~N}$ to $3247 \mathrm{~N}$. Hence, $\alpha=11.7^{\circ}$ is assumed as the final value of the angle $\alpha$. This value is taken as the limit because along with the lifting of the upper frame (bed deck) the angle increases, and the higher the initial value, the higher the end value of the angle. This is related to a considerable angular lift of the actuator body, which can greatly reduce the space under the bed deck at its maximum height. In accordance with the objectives, effort was taken to ensure the largest possible free space under the bed deck.

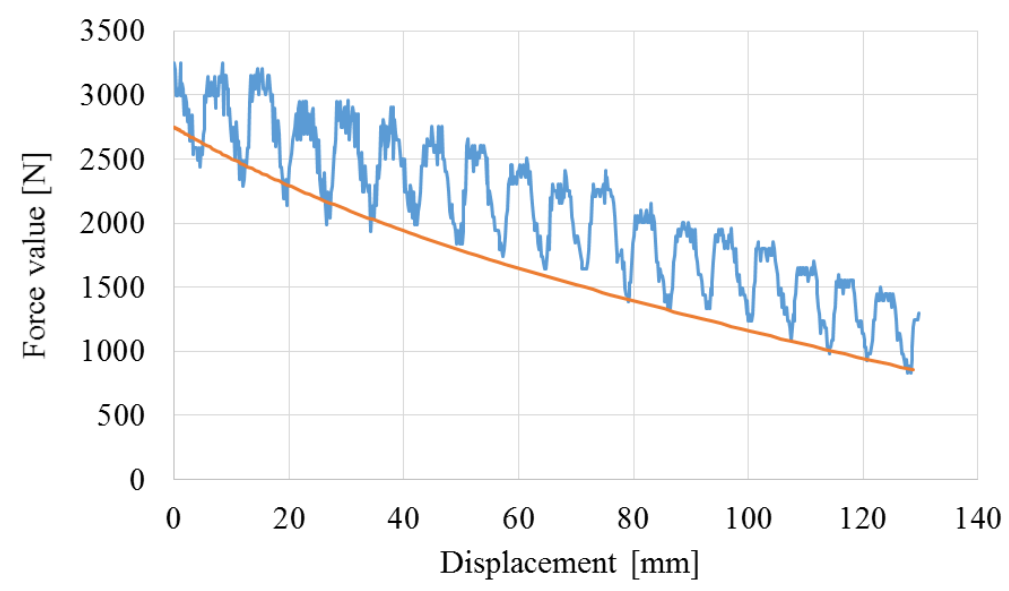

Fig. 11. Characterization comparison of the changes of the force $F$ acting on the axis of the hydraulic actuator rod needed to power the bed deck adjustment mechanism for the angle

$\alpha=11.7^{\circ}$ and the load corresponding to the patient's weight $\mathrm{m}=86 \mathrm{~kg}$, where: 1 - characterization obtained from simulation, 2 - characterization obtained from testing

Figure 12 presents the characterization comparison of the changes of the force $(\mathrm{F})$, taken from the simulation and the actual prototype tests, for the angle $\alpha=11.7^{\circ}$ and the bed deck load 12 (fig. 1a) corresponding to the load with the patient's weight $\mathrm{m}=160 \mathrm{~kg}$. 


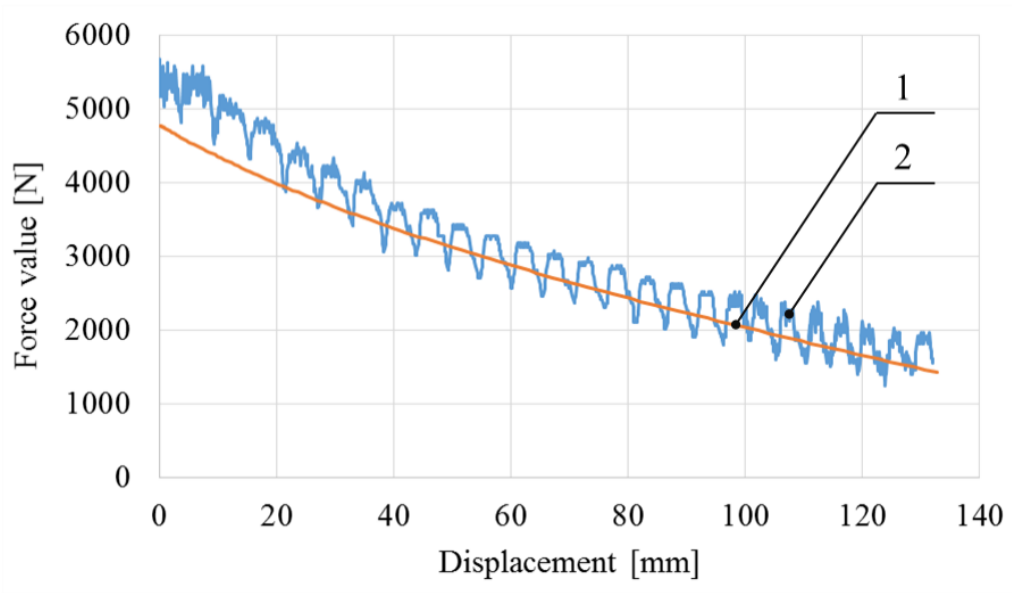

Fig. 12. Characterization comparison of the changes of the force $F$ acting on the axis of the hydraulic actuator rod needed to power the bed deck adjustment mechanism for the angle $\alpha=11.7^{\circ}$ and the load corresponding to the patient's weight $m=160 \mathrm{~kg}$, where:

1 - characterization obtained from simulation, 2 - characterization obtained from testing

For loading the upper frame of the bed prototype with the weight $\mathrm{m}=160 \mathrm{~kg}$ the maximum force $\mathrm{F}$ is $5679 \mathrm{~N}$. This result suggests it is feasible to use a hydraulic actuator with a smaller maximum force $(\mathrm{F}<10 \mathrm{kN})$, which means it is possible to purchase a cheaper actuator.

\section{Conclusions}

This work presented the design of the hospital bed deck height adjustment mechanism formulated on the basis of the specific design objectives. In accordance with the principles of concurrent engineering, the virtual model of the bed and its height adjustment mechanism was made and a kinematic analysis was performed. The results of this analysis made it possible to select appropriate geometric parameters of the lifting mechanism, which provides the required free space under the bed deck for diagnostic tests. The mechanism also allows bed deck height adjustment within the required range.

In the next stage, after the completion of the prototype build, it is planned to test the bed prototype in operating conditions of the hospital environment.

\section{References}

1. H. H. Nguyen, T. N. Nguyen, R. Clout, H. T. Nguyen, A novel target following solution for the electric powered hospital bed. Proceedings of the Annual International Conference of the IEEE Engineering in Medicine and Biology Society 7319164, 3569-3572, (EMBS 2015)

2. D. T. Johnson, P. D. Holtan, Bed transfer system. Patent US7533742 B2, 19 May (2009)

3. Thomas W. Hanson, R. S. Henderson, Combination bed mover and patient transfer apparatus. Patent US 6834402 B2, 28, (2004)

4. P. Shih-Wei, L. Feng-Li, F. Li-Chen, Mechanism Design and Mechatronic Control of a Multifunctional Test Bed for Bedridden Healthcare, Mechatronics, IEEE/ASME Transactions on 15, 234-241 (2010) 
5. C. Wang, A. V. Savkin, R. Clout, H. T. Nguyen, An intelligent robotic hospital bed for safe transportation of critical neurosurgery patients along crowded hospital corridors. IEEE Transactions on Neural Systems and Rehabilitation Engineering 23 (5), 6879265 , 744-754

6. N. Huy Hoang, N. Tuan Nghia, R. Clout, H. T. Nguyen, An advanced control strategy of an electrical - Powered hospital bed, in Engineering in Medicine and Biology Society (EMBC). $36^{\text {th }}$ Annual International Conference of the IEEE, (2014)

7. H. Wang, F. Kasagami, Careful-patient mover used for patient transfer in hospital. IEEE/ICME International Conference on Complex Medical Engineering, 20-26 (2007)

8. W. Ching-Hua, T. Ting-Chun, H. Shin-Chieh, C. Wan-Chun, C. Yen-Ming, T. KunTse, Y. Chun-Went, C. Kuo-Yi, Hospital bed with auxiliary functions of lateral positioning and transferring for immobilized patients. The $33^{\text {rd }}$ Annual Conference of the IEEE Industrial Electronics Society (IECON), 2991-2995 (2007)

9. A. Basmajian, E. Blanco, H. Asada, The marionette bed: automated rolling and repositioning of bedridden patients. in Proceedings of the 2002 IEEE International Conference on Robotics \& Automation, 1422-1427 (2002)

10. A. Andhare, A. Onkar, P. Padole, Design of bed for bedridden patients: Analysis and synthesis of mechanisms. $15^{\text {th }}$ National Conference on Machines and Mechanisms, NaCoMM (2011)

11. S. Mascaro, J. Spano, H. Asada, A reconfigurable holonomic omni-directional mobile bed with unified seating (RHOMBUS) for bedridden patients. IEEE International Conference on Robotics and Automation, 1277-1282 (1997)

12. L. Tan, S. Lu, W. Zhang, A robotic nursing bed design and its control system. Proceedings of the 2009 IEEE International Conference on Robotics and Biomimetics, December 19-23, (2009)

13. S. Peng, F. Lian, Mechanism design and mechatronic control of a multifunctional test bed for bedridden healthcare. IEEE/ASME Transactions on Mechatronics 15 (2), 234241 (2010) 